\title{
UV-VIS Spectrum
}

National Cancer Institute

\section{Source}

National Cancer Institute. UV-VIS Spectrum. NCI Thesaurus. Code C63858.

A spectrum that contains waveleng th values that correspond to ultraviolet (UV) and visible (VIS) light properties. 